\title{
ON THE (NON)-COINCIDENCE OF MILNOR-THURSTON HOMOLOGY THEORY WITH SINGULAR HOMOLOGY THEORY
}

\author{
ANDREAS ZASTROW
}

The paper investigates a homology theory based on the ideas of Milnor and Thurston that by considering measures on the set of all singular simplices one should get alternate possibilities for describing the cycles of classical homology theory. It suggests slight changes to Milnor's and Thurston's original definitions (giving differences for wild topological spaces only) which ensure that their homology theory is well-defined on all topological spaces. It further proves that Milnor-Thurston homology theory gives the same homology groups as the singular homology theory with real coefficients for all triangulable spaces. An example showing that the coincidence between these both homology theories does not hold for all topological spaces is also included.

The idea of the Milnor-Thurston homology is, roughly speaking, that the usual homology groups with real coefficients can be defined by considering measures on the set of all singular simplices when using appropriate definitions, and hence that infinite chains can be defined, which is of some use in certain proofs because of the additional possibilities that this homology theory provides in representing the cycles. Since those measures which are concentrated on a finite number of singular simplices give the same behavior as the corresponding formal sum of these singular simplices in the singular homology theory, the definition of chains by measures as explained in the first sentence can be regarded as a generalisation of the usual chains. The possibility that such a homology theory can by used in the proofs of the Mostow and Gromov Theorems for hyperbolic manifolds was stated in Thurston's famous preprint [Th] (pp. 6.6-6.7). Thurston there referred this idea to a joint work with Milnor and to a forthcoming paper of [MTh], which, however never appeared ([MR], 1977-1994). The use of this homology theory can be avoided in the proof of the Mostow Theorem, but its use is essential in a proof of the fact the three-dimensional closed oriented hyperbolic manifolds with the same volume are isometric, if they can be mapped onto each other by a degree-one mapping. This is the theorem we are calling "Gromov Theorem", since Thurston referred this statement 
to him ([Th], (Thm. 6.4)/[HM], (Thm.1)). Gromov in [Gr], $\S 2$ (last line) however circumvents the use of Milnor-Thurston homology theory and takes a dual point of view instead. Other traces of this homology theory can be found in the book of Benedetti and Petronio $[\mathbf{B P}]$, (page xii) (who explain in their introduction they will avoid using this theory) and in Munkholm's workout ([Mnkh], $(\S 4))$ of the corresponding paragraphs of [Th]. Neither Thurston nor Munkholm, however, do give much information on how to prove that this homology theory is well-defined and gives the usual homology groups (more can be found in Ratcliffe's book [Rcl], (Thm. 11.5.1), but cf. Remark 0.1 below). The purpose of this paper now is to give elaborated versions of these proofs while adapting the definitions as originally suggested by Milnor and Thurston so that they can be applied to a much larger class of spaces as the original ones. More precisely, the content of this paper can be characterized as workouts of the proofs of the following facts:

(i) We suggest for all topological spaces and all pairs of topological spaces the definition of some homology theory which has infinite chains defined by considering measures on the set of all singular simplices (cf. 1.8, 1.12, 1.13 and 2.9-2.10(ii)).

(ii) This theory gives for all (possibly infinite) simplicial complexes (in the relative case: Pairs of simplicial complexes) the same homology groups as the classical singular theory with real coefficients; a natural isomorphism is constructed (cf. Sect. 5).

(iii) For differentiable manifolds our definitions do not give other homology groups as Milnor's and Thurston's original definitions (cf. 3.4).

(iv) The coincidence in the sense of (ii) does not hold for all topological spaces. An example showing this fact is included in Section 6.

The outline of the coincidence proof is due to Eilenberg and Steenrod ([ES]) and can be interpreted as applying a standardized final conclusion after showing that the Milnor-Thurston homology theory satisfies several properties ("Eilenberg-Steenrod-Axioms"). So the main difficulties arise when trying to verify these axioms, since not all proof techniques of singular homology theory can be used in Milnor-Thurston theory also, and some can only be applied after having taken additional care. The crucial point can be seen as follows: In singular homology theory the set of singular simplices is a free basis and hence almost every construction which can be performed with singular simplices extends to homomorphisms between the singular chain groups. But in Milnor-Thurston homology theory the set of singular simplices can only be regarded as points of some space where only continuous mappings extend to homomorphisms of the Milnor-Thurston chain groups (cf. 2.1(i)). This continuity is not given in every case (e.g. 4.8-4.9/4.12), and even where it is given in singular homology one does not usually pay 
attention to it. Because of those phenomena this paper proves the excision theorem only under additional conditions for non-normal spaces (cf. 4.1).

0.1. Remark. It turns out that in the highest dimension of a closed oriented hyperbolic manifold the isomorphism between the two homology theories of consideration (cf. (ii) above) is an isometry with respect to the Gromov norm "\|l\|". Note that this property is essential for the desired application of this homology theory in the proofs of the Mostow and the Gromov Theorems. The original draft of this paper contained an additional seventh section proving also that this isometry exists. The proof was based on showing that

(1) $\operatorname{Vol}(M) /\|[M]\|$ is the same constant given by the volume of the ideal regular $n$-simplex,

regardless whether we define the Gromov Norm using Milnor-Thurston or classical homology theory, (cf. [Th], (Def. 6.1.8), [Th], (Def. 6.1.2), respectively). We took this section out, since meanwhile there is a shorter proof available in standard literature. The proof of (1) for singular homology theory can be found in $[\mathbf{B P}]$, (Thm. C.4.2) while [Rcl], (Thm. 11.5.4) gives the key lemma for transforming the proof to Milnor-Thurston homology theory. However, in his exposition Ratcliffe follows the original definitions of Milnor and Thurston and makes full use of the advantages of differential geometry.

Acknowlegdement. I thank Prof. Boileau for helpful discussions.

\section{Definition of the Milnor-Thurston homology groups.}

1.1. General Remarks. The Milnor-Thurston homology groups are obtained according to the usual definition scheme from a special chain complex, hence the construction of the chain complex is the first topic of this paper. We will use the script letters $\mathcal{C}, \mathcal{Z}, \mathcal{B}$ and $\mathcal{H}$ to denote the chain-, cycle, boundary-, and homology-groups of the Milnor-Thurston homology, respectively, whereas the same letters in the ordinary typeface denote the corresponding groups of the singular homology theory. Since in this paper the singular homology theory with real coefficients is compared with the Milnor-Thurston homology theory, we define $H_{k}(X):=H_{k}(X ; \mathbb{R})$, i.e. we agree that for the singular homology theory the domain of coefficients shall be the real numbers in this paper, if not explicitly denoted. Even if most of the chain and homology groups to be considered in this paper are vector-spaces by definition, we will usually call them "groups".

1.2. Some Remarks on Measures. The measures to be considered in this context are usually signed measures, i.e. measures which can take negative values as well. Hence a "measure on a set $X$ " is in our context defined to be a function that assigns to each set of a sigma-algebra $\mathbb{A}$ of subsets of $X$ a 
real number or $\pm \infty$ such that this function is additive and sigma-additive. This paper restricts to considering measures defined on topological spaces where $\mathbb{A}$ is in every case the Borel-sigma-algebra $\mathbb{B}$, i.e. the smallest family of sets containing all open sets which is closed with respect to complements and finite or countable union of sets. These Borel-sets are identified with the measurable sets in this paper. The (sigma-)additivity of the measures requires that the measure of a finite or countable union of pairwise disjoint measurable sets can be calculated by taking the sum of the measures of the sets. It also implies that no measure can be allowed to take on both of the values $+\infty$ and $-\infty$. Hence the sum of two measures which have $+\infty$ and $-\infty$ as values respectively cannot be defined to be a measure. On the other hand sums and real multiples of measures taking on finite values only are always well-defined. Hence the set of these measures has the structure of a real vector space. This structure is used when defining the Milnor-Thurston homology groups.

1.3. Some Notation for Measures. Recall that the basic separation theorem ([Hal], VI.§29 (Thm. A\&B) or [Hen], 5.1 (Satz 4), cf. also [Bau2], II.§18) for signed measures tells us that each signed measure $\mu$ can be uniquely decomposed as the difference $\mu=\mu^{+}-\mu^{-}$of two positive measures (i.e. measures taking on no negative values). This decomposition is unique, given the additional requirement that there exists a (non-unique) decomposition $X=X^{+} \cup X^{-}$of the basic space $X$ into two measurable sets such that each of the two decomposing measures $\mu^{+}, \mu^{-}$takes the value zero on all subsets of the decomposition spaces indexed by the opposite sign. $\mu^{+}$and $\mu^{-}$are called the "positve/negative part of $\mu$ " or the "upper/lower variation". Do not confuse the "absolute value" of a measure (which is the measure $|\mu|:=\mu^{+}+\mu^{-}$and is also called "total variation") with the "norm" of a measure, which is the non-negative real number (or " $\infty$ ") defined by $\|\mu\|:=\max _{A \in \mathbb{B}(X)} \mu(A)-\min _{B \in \mathbb{B}(X)} \mu(B)=|\mu|(X)$. Note that this norm satisfies the usual triangle inequality, as one can first prove straightforwardly for positive measures and then extend to signed measures. The extension step is also straightforward if one has observed that a sum-relation for three signed measures (" $\mu_{1}+\mu_{2}=\nu$ ") can only be turned into corresponding relations for the positive or negative part by adding a correction summand $\nu_{1}$ or $\nu_{2}$ for each sign according to " $\mu_{1}^{ \pm}+\mu_{2}^{ \pm}=\nu^{ \pm}+\nu_{1 / 2}$ ", but that these correction summands turn out to be the same positive measure.

\subsection{Two special types of measures.}

(i) If the letter $\mu$ is indexed by a point lying in some space $X$, this gives our standard notation for the "atomic measure" defined on $X$ :

$$
\mu_{P}(A):= \begin{cases}1 & \text { if } P \in A, \\ 0 & \text { if } P \notin A .\end{cases}
$$


(ii) Measures which are concentrated on a finite number of points are regarded as "counting measures". Such measures are usually described as linear combinations of atomic measures.

(iii) Remark. Let $\nu=\sum_{i=1}^{n} \alpha_{i} \cdot \mu_{P_{i}}$ be a finite counting measure such that $P_{1}, \ldots, P_{n}$ are mutually disjoint. Then $\|\mu\|=\sum_{i=1}^{n}\left|\alpha_{i}\right|$ is straightforward from the definitions.

\subsection{Further notation for measures.}

(i) Let $f: X \rightarrow Y$ be a continuous mapping and let $\mu$ be a measure on $X$. Then $f(\mu)$ denotes the measure defined on $Y$ by $(f(\mu))(A):=$ $\mu\left(f^{-1}(A)\right)$ for any measurable $A \subset Y$. The continuity of $f$ and our restriction to measures defined on the Borel-sigma-algebra guarantee that this construction of an "image measure" is well-defined.

(ii) Finally we define $\operatorname{supp}(\mu)$ (the "support" of $\mu$ ) as the subset of $X$ obtained as the set of all points of $X$ which have no open neighbourhood that is a zero-set of $\mu$. Note that the zero-sets of signed measures have to be more carefully characterized than for positive measures: The conditions " $\mu(B)=0$ for any subset $B$ of $A$ " and " $|\mu|(A)=0$ " are sufficient, but " $\mu(A)=0$ " does not imply that " $A$ is a zero-set of $\mu$ ".

Since in general spaces the complement of the support of a measure need not be a zero-set (cf. 3.1), we need the following:

\subsection{Definition.}

(i) A "determination set" of $\mu$ (where $\mu$ is a measure on $X$ ) is any (not necessarily measurable) subset of $X$ which satisfies that any (measurable) subset of its complement is a zero-set with respect to $\mu$.

(ii) If the support of a measure is a determination set, we call the support "proper".

\subsection{Definition.}

Let $\mu$ be a measure on a set of singular simplices and use $\sigma$ as the standard notation for one of those simplices. If such a measure has a proper support, we will call $\bigcup_{\sigma \in \operatorname{supp}(\mu)} \operatorname{Im}(\sigma)$ the "image of the measure $\mu$ " and denote it by $" \operatorname{Im}(\mu)$ "; if the support is not proper we fix a determination set $D$ of $\mu$ and will regard the non-uniquely defined set $\bigcup_{\sigma \in D} \operatorname{Im}(\sigma)$ similarly as the image of $\mu$. Do not confuse this term with the "image measure" as defined in $1.5(\mathrm{i})$ !

Now we are ready for the: 


\subsection{Definition of the absolute Milnor-Thurston chain-groups.}

$\mathcal{C}_{k}(X):=\{\mu \mid \mu$ is a quasicompactly determined measure

$$
\text { on } \left.C^{0}\left(\Delta_{k}, X\right) \text {, with }\|\mu\|<\infty\right\} \text {. }
$$

In other words: The $k^{\text {th }}$ chain-group of the Milnor-Thurston homology theory of the topological space $X$ consists of all measures defined on the space of all $k$-dimensional singular simplices topologized by the compact-open topology, such that for each of those measures the norm is finite and there exists a determination set which is quasi-compact, i.e. which need not be Hausdorff but satisfies the Heine-Borel covering property.

Lemma 1.9. By the natural addition and scalar multiplication of measures the Milnor-Thurston chain groups acquire the structure of a real vectorspace.

Proof. Note that the condition " $\|\mu\|<\infty$ " is equivalent to the property that $\mu$ takes neither of the values $\pm \infty$ (cf. 1.3/1.2). Hence all measures can be added (cf. 1.2). Thus it remains to be proved that the sum of quasicompactly determined measures is again quasicompactly determined. But this follows from the observation that the union of the determination sets of two measures is a determination set for the sum of those measures.

In order to introduce the relative Milnor-Thurston homology groups we need the following lemmata on the extension of measures and the Borelsigma-algebra of subspaces.

Proposition 1.10. Let $W$ be an arbitrary subspace of a topological space $X$ topologized by the relative topology. Then $\mathbb{B}(W)$, the Borel-sigma-algebra of $W$ generated by its subspace topology, consists precisely of all intersections of the Borel-sets of $X$ with $W$, or, in formulae:

$$
\mathbb{B}(W)=W \cap \mathbb{B}(X):=\{W \cap A \mid A \in \mathbb{B}(X)\} .
$$

Remark. In principle this compatibility between the subspace topology and trace-constructions in measure theory (cf. [Hen], 1.5 (Satz 8), [Bau2], I. $\S 1$ (1.4), [Bau1], I.1 (1.4)) is a known fact. However, since its proof is not straightforward and cannot be found in all standard literature, we are going to outline it in the following:

One of the two inclusions to be proven is straightforward: Since the relative topology of $W$ consists by definition of the intersections between the open sets of $X$ on the one hand and $W$ on the other hand, $W \cap \mathbb{B}(X)$ is a sigma-algebra containing all open sets of $W$, and $\mathbb{B}(W)$ is by definition the minimal one. Hence $\mathbb{B}(W) \subset(W \cap \mathbb{B}(X))$. For seeing the opposite inclusion regard $\mathbb{B}(X)$ as "extension" of $W \cap \mathbb{B}(X)$ from $W$ to $X$ and regard $\mathbb{B}(W) \cup((X-W) \cap \mathbb{B}(X))$ as "extension" of $\mathbb{B}(W)$, where the union (" $\cup$ ") 
when used between families of sets is to be understood as $\mathcal{A} \cup \mathcal{B}:=\{A \cup B \mid$ $A \in \mathcal{A}, B \in \mathcal{B}\}$. Both "extensions" of our sigma-algebras turn out to be sigma-algebras on $X$ containing all open sets of $X$, and hence we get, using the minimality-property of $\mathbb{B}(X)$, that $\mathbb{B}(X) \subset \mathbb{B}(W) \cup((X-W) \cap \mathbb{B}(X))$. Now applying to both sides of this inclusion the operation " $W \cap \ldots$ " yields the desired result.

Lemma 1.11. Let $W$ be a subset of $X$ and $\mu$ a measure defined on $W$. Then by

$$
\nu(A):=\mu(A \cap W) \text { for all } A \in \mathbb{B}(X)
$$

a measure $\nu$ is defined which can be regarded as the natural extension of $\mu$ from $W$ to $X$ by zero. Each determination set of $\mu$ is also one of $\nu$ and the norms of $\mu$ and $\nu$ coincide.

Proof. $\nu$ is well-defined by its definition (1), even if $W$ is not measurable as a subset of $X$, since by 1.10 we have $\mathbb{B}(W)=W \cap \mathbb{B}(X)$. Note that in this case, i.e. if $W \notin \mathbb{B}(X)$, we have to distinguish between the measurability of sets as subsets of $X$ and as subsets of $W$. Nevertheless we get that all determination sets of $\mu$ are determination sets of $\nu$, too, since by 1.6(i) determination sets need not be measurable and by (1) it is obvious that $\nu$ can take only the value 0 on the complement of any determination set of $\mu$. Now $\|\mu\|=\|\nu\|$ follows from 1.3 because $\nu$ takes by definition the same values as $\mu$.

\subsection{Definition of the relative Milnor-Thurston chain groups.}

Let $W \subset X$. Then

$$
\mathcal{C}_{k}(X, W):=\mathcal{C}_{k}(X) / \mathcal{C}_{k}(W) .
$$

Here the inclusion $W \hookrightarrow X$ induces an inclusion $C^{0}\left(\Delta_{k}, W\right) \hookrightarrow C^{0}\left(\Delta_{k}, X\right)$ such that each measure $\in \mathcal{C}_{k}(W)$ can also be regarded as an element of $\mathcal{C}_{k}(X)$ by Lemma 1.11. Since the zero-measure is the only measure permitted on the empty set, for $W=\emptyset$ we get that $C^{0}\left(\Delta_{k}, W\right)=\emptyset$ and hence $\mathcal{C}_{k}(W)=\{0\}$ so that $\mathcal{C}_{k}(X, W)=\mathcal{C}_{k}(X)$. Thus absolute Milnor-Thurston chain groups are just a special case of the relative ones.

1.13. Remark. Having defined Milnor-Thurston chain groups we continue this paper by explaining how operators and proof-techniques can be extended from sigular homology theory to Milnor-Thurston theory. Based on these techniques we will in the second half of the following section be briefly able to define the boundary operator and the homology groups in MilnorThurston theory and to deduce the category theoretical properties of this theory. 


\section{The Basic Extension Theorem of the Milnor-Thurston Homology Theory.}

Theorem 2.1. Let $f_{i, j}$ be operators, which map singular simplices to other singular simplices such that they can be interpreted as continuous functions between the spaces $C^{0}\left(\Delta_{\text {? }}\right.$, ?) of singular simplices. Then

(i) Each of these operators $f_{i, j}: C^{0}\left(\Delta_{k_{i-1}}, X_{i-1}\right) \rightarrow C^{0}\left(\Delta_{k_{i}}, X_{i}\right)$ has a canonical extension to a homomorphism $f_{i, j}: \mathcal{C}_{k_{i-1}}\left(X_{i-1}\right) \rightarrow \mathcal{C}_{k_{i}}\left(X_{i}\right)$ between Milnor-Thurston chain groups.

(ii) Let $\alpha_{i, j} \in \mathbb{R}$. If

$$
\left(\sum_{i_{1}=1}^{n_{1}} \alpha_{1, i_{1}} \cdot f_{1, i_{1}}\right) \circ\left(\sum_{i_{2}=1}^{n_{2}} \alpha_{2, i_{2}} \cdot f_{2, i_{2}}\right) \circ \ldots \circ\left(\sum_{i_{k}=1}^{n_{k}} \alpha_{k, i_{k}} \cdot f_{k, i_{k}}\right)=0
$$

in singular homology theory, then the analogous product of the corresponding operators in Milnor-Thurston theory vanishes also.

The Proof is spread over 2.2-2.6.

Lemma 2.2. If $\nu$ is an image measure which is constructed with respect to a continuous mapping as the image of a quasicompactly determined measure having a finite norm, then $\nu$ also satisfies these properties.

Proof. As an immediate consequence of the definition we get that the image of a determination set of a measure is a determination set for the image measure. In this context we need not worry about the measurability of the image, since we did not require (cf. 1.6(i)) that determination sets are measurable. Since the image of a quasicompact set with respect to a continuous mapping is always quasicompact (cf. [vQ], (Satz 8.11) or [Will], ${ }^{1}$ Chap. 6 (Thm 17.7)) the compatibility of the image measure construction with respect to the property of being quasicompactly determined follows. Observe that, since $\nu$ takes on by definition only values that are also taken on by its preimage $\mu$, we get by 1.3 that

$$
\|\nu\| \leq\|\mu\| .
$$

Note that the case " $<$ " can occur, if $f$ maps a non-trivial domain of $X^{+}$to the same set as some other domain of $X^{-}$.

2.3. Remark. The situation considered in Lemma 1.11 is just a special case of the situation considered now in Lemma 2.2: The extension of a measure is nothing but the image measure with respect to the inclusion map. The general case $f: X \rightarrow Y$ of Lemma 2.2 is in some sense easier,

\footnotetext{
${ }^{1}$ Note that Willard's use of the term "compact" corresponds to our and Von Querenburg's "quasicompact".
} 
because one need not distinguish between two concepts of measurability defined on the same space.

Lemma 2.4. Any continuous mapping $f: C^{0}\left(\Delta_{k}, X\right) \rightarrow C^{0}\left(\Delta_{m}, Y\right)$ extends to a homomorphism $f_{\bullet}: \mathcal{C}_{k}(X) \rightarrow \mathcal{C}_{m}(Y)$.

Proof. For each $\mu \in \mathcal{C}_{k}(X)$ we define $f_{\bullet}$ by

$$
f_{\bullet}(\mu)=f(\mu),
$$

where $f$ on the right hand side of this equation is to be considered as image measure construction in the sense of 1.5(i). Hence we will usually not distinguish between $f_{\bullet}$ and $f$ in this paper, i.e. we will usually drop the "().". Observe that the Milnor-Thurston chain groups do not contain all measures, but Lemma 2.2 guarentees that the image measure construction is compatible with the properties that characterize those measures that are elements of Milnor-Thurston chain groups. $f_{\bullet}$ is a homomorphism (i.e. a linear mapping), since

$$
f(a \cdot \mu+b \cdot \nu)=a \cdot f(\mu)+b \cdot f(\nu)
$$

is immediate from the definition 1.5(i) of image measures for all measures $\mu, \nu$ and $a, b \in \mathbb{R}$.

Lemma 2.4 gives the proof of Theorem 2.1(i). The next lemma is the first step of the proof of 2.1(ii), that permits restricting oneself to considering linear combinations instead of compositions of linear combinations as in 2.1(1).

Lemma 2.5. Let $f_{i, j}$ and $\alpha_{i, j}$ as in 2.1. Then we have

$$
\begin{aligned}
& \left(\sum_{i_{1}=1}^{n_{1}} \alpha_{1, i_{1}} \cdot f_{1, i_{1}}\right) \circ\left(\sum_{i_{2}=1}^{n_{2}} \alpha_{2, i_{2}} \cdot f_{2, i_{2}}\right) \circ \ldots \circ\left(\sum_{i_{k}=1}^{n_{k}} \alpha_{k, i_{k}} \cdot f_{k, i_{k}}\right) \\
& =\sum_{\left(i_{1}, \ldots, i_{k}\right) \in\left\{1, \ldots, n_{1}\right\} \times \ldots \times\left\{1, \ldots, n_{k}\right\}} \alpha_{1, i_{1}} \cdot \alpha_{2, i_{2}} \cdot \ldots \cdot \alpha_{k, i_{k}} \cdot\left(f_{1, i_{1}} \circ \ldots \circ f_{k, i_{k}}\right)
\end{aligned}
$$

in Milnor-Thurston theory and singular homology theory.

Proof. This equation follows immediately by using an arbitrary chain as argument and applying the linearity of the extension of the $f_{i, j}$ to singular or Milnor-Thurston chain groups successively. Cf. 2.4(2).

\subsection{Completion of the Proof of Theorem 2.1.}

By 2.5 it remains to be proved that if a simpler equation as 2.1(1), namely

$$
\sum_{i=1}^{k} \alpha_{i} \cdot f_{i}=0 \quad \text { with } \quad f_{i}: C^{0}\left(\Delta_{m}, X\right) \rightarrow C^{0}\left(\Delta_{n}, Y\right) \forall i
$$

is satisfied in singular homology theory, we get the same equation in MilnorThurston theory. This can be seen as follows: 
Define the equivalence relations on the index set by

$$
i \sim j \Longleftrightarrow f_{i}(\sigma)=f_{j}(\sigma) \text {. }
$$

Since this definition might depend on the singular simplex $\sigma$, we might get more than one equivalence relation so that there might be no minimal one. However, since the index set is finite, there are only finitely many possible equivalence relations; consider all of them and assign to each of these the set of singular simplices $\sigma \in C^{0}\left(\Delta_{m}, X\right)$ to which this equivalence relation belongs. This defines a partition of $C^{0}\left(\Delta_{m}, X\right)$. The partition sets $P_{1}, \ldots, P_{N}$ might be not measurable, but, since there are only a finite number of them, it is by [Hal], III. $\S 16($ Ex. (2)-(3)) possible to enlarge the Borel-sigma-algebra $\mathbb{B}\left(C^{0}\left(\Delta_{m}, X\right)\right)$ with the partition sets to $\mathbb{A}\left(C^{0}\left(\Delta_{m}, X\right)\right)$ and to extend all measures from $\mathbb{B}\left(C^{0}\left(\Delta_{m}, X\right)\right)$ to $\mathbb{A}\left(C^{0}\left(\Delta_{m}, X\right)\right)$, so that in the remainder of this proof the partition sets can be treated as measurable sets. Then we argue as follows: Let $\sigma \in P_{\nu}(\nu \in\{1, \ldots, N\})$ and consider the corresponding equivalence relation $\sim_{\nu}$ defining a partition of the index set $\{1, \ldots, k\}$ into equivalence classes $Q_{\nu, 1}, \ldots, Q_{\nu, j_{\nu}}$. Now, use that by assumption according to (1) we have $\sum_{i=1}^{k} \alpha_{i} \cdot f_{i}(\sigma)=0$ for all singular simplices $\sigma$ in singular homology theory which implies that for all $\nu \in\{1, \ldots, N\}$ and all $\iota \in\left\{1, \ldots, j_{\nu}\right\}$ we have $\sum_{i \in Q_{\nu, \iota}} \alpha_{i}=0$ and $\left.f_{i}\right|_{P_{\nu}}=\left.f_{j}\right|_{P_{\nu}}$ for $i, j \in Q_{\nu, \iota}$. Now this implies that for each $P_{\nu}$ the image measure of the restriction to $P_{\nu}$ of an arbitrary measure $\mu$ with respect to $\sum_{i=1}^{k} \alpha_{i} \cdot f_{i}$ vanishes; and hence, since $\bigcup_{\nu=1}^{N} P_{\nu}$ covers $C^{0}\left(\Delta_{m}, X\right)$, we have $\sum_{i=1}^{k} \alpha_{i} \cdot f_{i}=0$ for all measures defined on $C^{0}\left(\Delta_{m}, X\right)$ which is the desired result.

2.7. Remark. For the sake of having a smoothly applicable theorem, we had been discussing the most general situation in the proof of 2.6 , even if none of the cases where we want to apply this theorem is as general as the one discussed: In the standard situation from considering unsymmetric singular simplices one gets an equivalence relation that is minimal in the sense that it only relates two elements to each other if any other equivalence relation as constructed by 2.6 does the same. Then by using that relation, in the proof of 2.6 the split-up into possibly non-measurable partition sets could have been skipped.

For the applications of the above Theorem 2.1 the following three facts are useful:

\section{Lemma 2.8.}

(i) Let $f: X \rightarrow Y$ be continuous. Then $C^{0}\left(\Delta_{k}, X\right) \longrightarrow C^{0}\left(\Delta_{k}, Y\right), \sigma \longmapsto$ $f \circ \sigma$ is continuous.

(ii) Fix $\tau \in C^{0}\left(\Delta_{m}, \Delta_{k}\right)$. Then $C^{0}\left(\Delta_{k}, X\right) \rightarrow C^{0}\left(\Delta_{m}, X\right), \sigma \mapsto \sigma \circ \tau$ is continuous.

(iii) $C^{0}\left(\Delta_{k}, X\right) \longrightarrow C^{0}\left(\Delta_{k} \times I, X \times I\right), \sigma \longmapsto \sigma \times \operatorname{id}_{I}$ is continuous. 
Proof. By using the fact that the topology established on the spaces $C^{0}(\cdot, \cdot)$ is the compact-open one the three above statements all follow from set topological conclusions.

Corollary 2.9. The extension of the boundary operator $\partial$ of singular homology theory to Milnor-Thurston theory in the sense of $2.1(\mathrm{i}) / 2.4$ gives the boundary operator $\partial$ in Milnor-Thurston theory. This extension also satisfies $\partial^{2}=0$. Recall that in singular theory the boundary operator is defined as $\partial:=\sum_{i=0}^{k}(-1)^{k} \delta_{i}$ with $\sigma \mapsto \sum_{i=0}^{k}(-1)^{k}\left(\sigma \circ \delta_{i}\right)$, where

$$
\delta_{i}: \Delta_{k-1}=\left[P_{0}, P_{1}, \ldots, P_{k-1}\right] \rightarrow\left[P_{0}, P_{1}, \ldots, P_{k}\right]=\Delta_{k}
$$

is the affine mapping obtained by extending the definition $P_{j} \mapsto P_{j}$ for $i<j$ and $P_{j} \mapsto P_{j+1}$ for $j \geq i$. Hence by 2.8(ii) and 2.1(i) $\partial$ can be extended to Milnor-Thurston theory and the extension satisfies $\partial^{2}=0$ by 2.1(ii).

We now merely need to quote the above extension facilities and standard arguments of homology theory to obtain the basic concepts and properties of a homology theory in the Milnor-Thurston-case, thus:

\subsection{Lemma-Definition.}

(i) Let $X$ be a topological space and let $W$ be an arbitrary subset (cf. 1.12). Since by the naturality of their definitions the boundary operators (cf. 2.9) of $\mathcal{C}_{*}(X)$ and $\mathcal{C}_{*}(W)$ are compatible with the inclusion $\mathcal{C}_{*}(W) \hookrightarrow \mathcal{C}_{*}(X)$, a boundary operator $\partial$ satisfying $\partial^{2}=0$ is induced on the relative Milnor-Thurston chain groups, too.

(ii) Relative and absolute ( $W=\emptyset$, cf. 1.12) Milnor-Thurston cycle, boundary, and homology groups can now be defined following the standard scheme: $\mathcal{Z}_{k}(X, W):=\operatorname{ker}\left(\mathcal{C}_{k}(X, W) \stackrel{\partial}{\longrightarrow} \mathcal{C}_{k-1}(X, W)\right) \mathcal{B}_{k}(X, W):=$ $\operatorname{Im}\left(\mathcal{C}_{k+1}(X, W) \stackrel{\partial}{\longrightarrow} \mathcal{C}_{k}(X, W)\right), \mathcal{H}_{k}(X, W):=\mathcal{Z}_{k}(X, W) / \mathcal{B}_{k}(X, W)$.

(iii) Any continuous map between pairs of topological spaces $f:(X, W) \rightarrow$ $(Y, V)$ naturally induces a chain mapping between Milnor-Thurston complexes $\mathcal{C}_{*}(X, W) \rightarrow C_{*}(Y, V)$, similarly in the absolute case.

(iv) Given the same assumptions as in 2.10(iii), on the level of homology induced homomorphisms $f_{*_{n}}: \mathcal{H}_{n}(X, W) \rightarrow \mathcal{H}_{n}(Y, V)$ are defined.

(v) These homomorphisms satisfy: $(f \circ g)_{*_{n}}=f_{*_{n}} \circ g_{*_{n}}$, and $\mathrm{id}_{*_{n}}=\mathrm{id}$ for all $n \in \mathbb{N}_{0}$, whenever $f$ and $g$ are continuous composible mappings between pairs of topological spaces.

(vi) Let $f \simeq g:(X, W) \rightarrow(Y, V)$. Then $f_{*_{n}}=g_{*_{n}}$ for all $n \in \mathbb{N}_{0}$ (for a proof see 5.3).

As can be seen from the standard argument of applying 2.10(vi) and 2.10 (v) to the composition of $f$ and its homotopy inverse, we have that

(vii) if $f$ is a homotopy equivalence, $f_{*_{n}}$ is an isomorphism.

Finally, 2.10(ii)-2.10(vii) can be summed up as follows: 
(viii) The construction of the $n^{\text {th }}$ Milnor-Thurston homology groups can be regarded as a covariant homotopy functor which has the category of topological spaces or pairs of topological spaces as its domain and the category of real vector spaces as its codomain.

Proof - Ad 2.10(iii). In the absolute case $f$ induces a continuous mapping $C^{0}\left(\Delta_{k}, X\right) \rightarrow C^{0}\left(\Delta_{k}, Y\right), \sigma \mapsto f \circ \sigma$ which by 2.1(i) yields induced homomorphisms $f_{\bullet}$ for Milnor-Thurton chain groups. Since the operations $\sigma \mapsto f \circ \sigma$ and $\sigma \mapsto \sigma \circ \delta_{i}$ (cf. 2.9) naturally commute, we get $f_{\bullet_{n-1}} \circ \partial=\partial \circ f_{\bullet_{n}}$, i.e. that $f_{\bullet}$ is a chain map. In the relative case one must in addition observe that the above processes commute with the natural inclusions $W \hookrightarrow X$ and $V \hookrightarrow Y$.

Ad 2.10(iv). Use 2.10(iii) to obtain induced mappings on the chain level, and then standard conclusions of homological algebra (e.g. [StZ], III.8.3.4$[\mathbf{S t Z}]$, III.8.3.5/[Sp], 4.1.1) to get induced homomorphisms on the level of homology groups as well.

Ad 2.10(v). These relations are, step by step, deduced on the various levels, using on the one hand standard conclusions of homological algebra, and on the other hand Theorem 2.1(ii) for passing from singular chain groups to Milnor-Thurston chain groups.

\section{On the basic ideas of the definitions of this homology theory, as originally stated and as used in this paper.}

\subsection{On some of the reasons for our changing of the original defi- nitions.}

As mentioned before, in order to get a well-defined homology theory on arbitrary topological spaces, the definitions as originally suggested by Milnor and Thurston in [Th], (pp. 6.6-6.7) had to be changed a bit, the most obvious need for a change being imposed by their use of differential geometry. Milnor and Thurston consider differentiable singular simplices only, and metricize the corresponding space by the $C^{1}$-topology. This enabled them to use the powerful tools of differential geometry (e.g. differential forms and duality) in their arguments; but, of course, this restricts the application of this theory to spaces which have an underlying differentiable structure. Since we do not assume a differentiable or even a metric structure, we have little other choice than to consider all singular simplices and to use in 1.8 the compact-open topology on $C^{0}\left(\Delta_{?}\right.$, ?)-spaces. Further adaptation of definition was necessary, since the concept of considering compactly supported measures does not work for arbitrary topological spaces. For, the concept of compactness requires the Hausdorff property, and so this can cause unwanted obstructions for the existence of compact supports in non-Hausdorff spaces. Hence in 1.8 we have to work with the concept of quasicompactness 
instead. Further note that since the classical defining condition for a support only requires that each point in the complement has a neighbourhood with measure zero, for spaces which do not have a countable topological basis we cannot rule out that there exist non-zero sets in the complement of the support of some measure. This in particular could result in the support of the image measure for a projection-like map being bigger than the image of the support of a measure. For measures with infinite norm such examples can explicitly be constructed. As a third point note that, when we have " $<"$ in $2.2(1)$ and $\operatorname{supp}(f(\mu))_{\neq}^{\subset} f(\operatorname{supp}(\mu))$, for sufficiently wild topologies we cannot rule out phenomena as described in the previous sentence for $f(\operatorname{supp}(\mu))-\operatorname{supp}(f(\mu))$, even if the support of $f$ is proper. This makes it desirable to work with non-uniquely defined determination sets. However, for non-pathological sets one need not worry about some of the difficulties described above, because one has:

Lemma 3.2. Let $Y$ be a metric separable space and let $\mu$ be a measure on $C^{0}\left(\Delta_{k}, Y\right)$. Then $\mu$ has a quasicompact determination set, if and only if its support is compact and proper. In such a case the support is the smallest determination set.

Proof. Since by the Definitions 1.5(ii)/1.6 each compact proper support gives a quasicompact determination set also, "£" is trivial; hence in the follow-

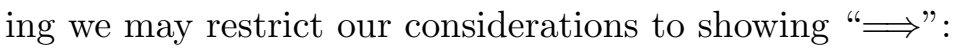

Let $\mu$ be a measure and let $D$ be a quasicompact determination set for $\mu$. Define $S:=\operatorname{supp}(\mu)$, so that we are left with showing that $S$ is compact, proper and contained in $D$. Now the complement of $S$ satisfies by its Definition 1.5(ii) that each point has at least one neighbourhood which is a zeroset. Since $Y$ is by assumption metric and separable, it has a countable topological basis (cf. [Dug], IX.5.6). The property of being metric and separable is passed on from $Y$ to $C^{0}\left(\Delta_{k}, Y\right)$, since by [Dug], XII.8.2(3)/[Dug], XII.1.1 the supremum metric induces the compact-open topology of $C^{0}\left(\Delta_{k}, Y\right)$, and then [Dug], XII.5.2 gives a countable topological basis for $C^{0}\left(\Delta_{k}, Y\right)$, also. Hence the complement of $S$ can be described as a countable union of open zero-sets, which gives that it is a zero-set itself. Thus $S$ is proper. Now assume there exists a point $P$ with $P \in S$, but $P \notin D$. Recall that the topology of $Y$ or $C^{0}\left(\Delta_{k}, Y\right)$ is metric and hence Hausdorff, so that the concepts of compactness and quasicompactness coincide. Hence $D$ is compact and thus closed, so that $P$ has an open neighbourhood $U(P)$ with $U(P) \cap D=\emptyset$. Since $P \in S$, this $U(P)$ cannot be a zero-set which contradicts to $D$ being a determination set. Hence there is no such $P$ which implies that $S \subset D$. Thus $S$ which by definition is closed occurs as a subset of the compact set $D$, and hence it is compact, also. This completes the proof.

As a consequence of 3.2 we get: 
Corollary 3.3. All elements of the Milnor-Thurston chain groups $\mathcal{C}_{k}(X)$ have a unique image, if $X$ is metric and separable (cf. 1.7/1.8).

Theorem 3.4. For any differentiable manifold the Milnor-Thurston homology groups as defined in this paper coincide via natural isomorphisms with the ones defined by the original scheme of Milnor and Thurston that will be denoted by " $\mathcal{H}_{k}^{\text {diff" }}$ (cf. 3.1).

Proof. There might exist proofs of this theorem that are based on using the powerful techniques of differentiable manifolds; however, since $[\mathbf{R c l}], \S 11.5$ does not contain such a proof (in fact, [Rcl], (Thm. 11.5.1) only gives that the concatenation of the desired natural map with some other map induces isomorphisms in homology), in our situation the shortest way for obtaining this theorem appears to be to prove each of the three subsequent isomorphy relations independently for any differentiable manifold $M$ by using the methods as developed in this paper:

$$
\mathcal{H}_{k}(M) \stackrel{(1)}{\cong} H_{k}(M) \stackrel{(2)}{\cong} H_{k}^{\text {diff }}(M) \stackrel{(3)}{\cong} \mathcal{H}_{k}^{\text {diff }}(M)
$$

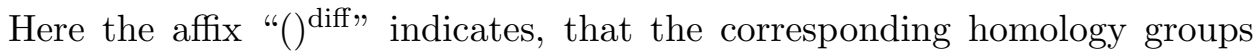
are to be computed by considering only differentiable mappings as singular simplices. The groups " $H_{k}^{\text {diff" }}$ are often considered in differential geometry, and (2) is standard knowledge of that theory. The relation (1) is our coincidence Theorem 5.0, and (3) is what remains to be proved and what can be

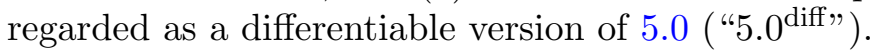

In order to obtain the corresponding statement we can use an argument obtained by substituting the objects of consideration in the series of definitions and lemmata in this paper correspondingly. In most cases these substitutions only cause natural adaptations in the arguments in the corresponding proof. In the following we give a guide for the substitutions that are necessary to turn the definitions, statements and proofs of this paper into a line of arguments that give " 5.0 diff":

The basic objects of consideration have to be differentiable manifolds $M, N, \ldots$ instead of topological spaces $X, Y, \ldots$. Where in Section 5 our consideration will be restricted to simplicial complexes, for obtaining $5.0^{\text {diff }}$ we will have to require that these manifolds are equipped with a triangulation, the existence of which is guaranteed by [Mnkr], (Thm. 10.6). In the adapted version we need not consider arbitrary subspaces any longer, but we may restrict our consideration to submanifolds that fit into the triangulation of a given manifold. When adapting our proof, all spaces of type $C^{0}\left(\Delta_{?}, X\right)$ have to be replaced by spaces $C^{1}\left(\Delta_{?}, M\right)$ and the corresponding compact-open topology has to be replaced by a $C^{1}$-topology, i.e. by a topology induced by a metric that results from adding to the standard supremum metric of our function the suprema of all first partial derivatives. Correspondingly all singular simplices that need to be considered in the adapted 
version are differentiable. We also need to require such differentiability for all mappings and homotopies between the manifolds $M, N, \ldots$ of consideration, even if for the ordinary version of 5.0 plain continuity is sufficient. This differentiability usually causes just standard adaptations in the proofs, since for most of the lemmata the additional requirement of differentiability affects the assumptions and the conclusions of the lemmata simultaneously. There are some minor exceptions (i.e. 4.4 and 5.3), where it takes additional straightforward verifications that some singular simplices that are explicitly constructed in the course of the proof turn out to be differentiable.

On the other hand, where continuous maps between spaces of type $C^{0}\left(\Delta_{?}, X\right)$ have to be considered, even the adapted version also requires continuity for the corresponding maps between the $C^{1}\left(\Delta_{?}, M\right)$-spaces, only. Due to these adaptations instead of results for the groups $\mathcal{C}_{?}(X), \mathcal{Z}_{?}(X)$ and $\mathcal{H}_{?}(X)$ we then get results for the groups $\mathcal{C}_{?}^{\text {diff }}(X), \mathcal{Z}_{?}^{\text {diff }}(X)$ and $\mathcal{H}_{?}^{\text {diff }}(X)$; wherever those groups occur they have to be substituted correspondingly. However, even if the substitutions as described so far are in general all fairly straightforward, our attention should now be drawn to those places where in proofs the compact-open topology of the spaces $C^{0}\left(\Delta_{?}, X\right)$ was explicitly referred to, since the topology of the spaces $C^{1}\left(\Delta_{k}, M\right)$ to be considered in the adapted version is to be defined in a different way. However, a sufficiently careful analysis gives that there are some places where only such standard properties of the $C^{0}$-topology are used, that also hold for the $C^{1}$-topology (as in 2.8 or $4.5(1)$ ), and some other places where it is sufficient to know that the $C^{1}$-topology is a refinement of the $C^{0}$-topology (as in 4.6) and apart from that there is only one exception where the following observation is of help:

If we replace a differentiable function $f: \Delta_{k} \rightarrow \mathbb{R}^{n}$ by a $(k \cdot n+1)$ tuple that comprises $f$ and all its partial derivatives ( $\left(f, \frac{\partial f}{\partial x_{1}}, \ldots, \frac{\partial f_{n}}{\partial x_{k}}\right)$ "), we can bijectively associate $\left\{f \mid f: \Delta_{k} \stackrel{\text { diff. }}{\longrightarrow} \mathbb{R}^{n}\right\}$ with a certain set of tuples of functions, or equivalently, with certain functions $\Delta_{k} \rightarrow \mathbb{R}^{n^{2} \cdot k+n}$. Now the $C^{0}$-topology of that set precisely coincides with the $C^{1}$-topology on the set $\left\{f \mid f: \Delta_{k} \stackrel{\text { diff. }}{\longrightarrow} \mathbb{R}^{n}\right\}$. From this observation and the fact that each manifold $M$ can be embedded into $\mathbb{R}^{m}$ for sufficiently big $m$, we get that a reference to [Dug], XII.5.2 can be used the same way in a proof of 5.0 diff as an argument to obtain that $C^{1}\left(\Delta_{k}, M\right)$ satisfies the second countability axiom, as a similar quoting of [Dug], XII.5.2 in the course of the proof of the ordinary coincidence Theorem 5.0 gave the analogous property for $C^{0}\left(\Delta_{k}, X\right)$ in 3.2 .

That gives us $5.0^{\text {diff }}$ and by $(*)$ completes the proof of this theorem. Of course a paper directly aiming for $5.0^{\text {diff }}$ could have abbreviated the proof in various places; in particular observe that our exceptional situation 3.2/3.3 is always given when considering differentiable manifolds. 


\section{The Excision Theorem.}

As mentioned in the introduction, we will prove the excision theorem of the Milnor-Thurston homology theory only under weaker assumptions than hold in the usual theory (see, for instance, [StZ], III.9.4.5 or [Sp], 4.6.5), namely:

Theorem 4.1. Let $U \subset W \subset X$ and assume there exists $V$ with

$$
\bar{U} \subset \stackrel{\circ}{V} \subset \bar{V} \subset \stackrel{\circ}{W} \text {. }
$$

Then $\mathcal{H}_{k}(X, W)=\mathcal{H}_{k}(X-U, W-U)$ for all $k \in \mathbb{N}$.

Remark. The proof of this theorem is split up into several lemmata presented below and is completed in 4.11. It follows the basic ideas of the proof in the singular homology theory, i.e. the use of barycentric subdivision in order to split up each cycle of $\mathcal{Z}_{k}(X)$ into two summands lying in $\mathcal{Z}_{k}(X-U)$ and in $\mathcal{Z}_{k}(W)$ so that the latter summand is trivial relative to $W$ (cf. [StZ], III.9.4.1-III.9.4.4 or [Sp], 4.4.10-4.4.14 and [Sp], 4.6.3-4.6.5). Hence the first few of the following lemmata are used to establish barycentric subdivision in the Milnor-Thurston theory:

\subsection{Definition of the barycentric operator $b_{n}: \mathcal{C}_{n}(X) \rightarrow \mathcal{C}_{n}(X)$.}

In principal this definition is obtained by extending the definition of this operator in singular homology theory using Theorem 2.1. Hence we start by recalling the standard construction of the barycentric operator: Let us first establish our notation for the standard unit simplex $\Delta_{n}$. We will use $P_{0}, \ldots, P_{n}$ to denote the vertices, $P$ as the name of its barycentre, and $\widetilde{P}_{i_{0}, \ldots, i_{k}}$ as notation for the barycentres of the lower-dimensional sides of $\Delta_{n}$. More precisely: $\widetilde{P}_{i_{0}, \ldots, i_{k}}$ is the barycentre of the $(n-k-1)$-dimensional side which is spanned by the vertices $P_{\text {? }}$ whose indices are not contained in the list $i_{0}, \ldots, i_{k}$. Now, for each permutation $\pi \in \mathcal{S}_{n+1}$ (i.e. $\pi$ bijects $\{0, \ldots, n\}$ onto itself) define $\beta_{n, \pi}: \Delta_{n} \rightarrow \Delta_{n}$ as the affine mapping obtained by extending $P_{0} \mapsto P, P_{i} \mapsto \widetilde{P}_{\pi(0), \ldots, \pi(i-1)}$, and let the chain $c_{\beta, n} \in C_{n}\left(\Delta_{n}\right)$ be $\sum_{\pi \in \mathcal{S}_{n+1}} \operatorname{sign}(\pi) \cdot \beta_{\pi, n}$, where " $\beta$ ", when used as an index, is to be regarded as a symbol. Then the $n^{\text {th }}$ barycentric operator of singular homology theory can be defined as the homomorphism $b_{n}: C_{n}(X) \rightarrow C_{n}(X), \sigma \mapsto \sigma_{\bullet}\left(c_{\beta, n}\right)$, where $\sigma \in C_{n}(X)$ is regarded as a mapping $\Delta_{n} \rightarrow X$ inducing a homomorphism $\sigma_{\bullet}: C_{n}\left(\Delta_{n}\right) \rightarrow C_{n}(X)$. By Theorem 2.1(i) this homomorphism $b_{n}$ extends to Milnor-Thurston chain groups, because the main assumption of Theorem 2.1, the continuity of the operation $\sigma \mapsto \sigma \circ \tau$ (where $\tau$ denotes one of the singular simplices the chain $c_{\beta, n}$ consists of), is given by Lemma 2.8(ii).

Corollary 4.3. Note that the barycentric operators as defined above form a chain map (i.e. we have $b_{n-1} \circ \partial_{n}=\partial_{n} \circ b_{n}$ for all $n$ ). 
This follows as an immediate application of Theorem 2.1(ii), since by 4.2 and 2.9 these operations are extensions in the sense of 2.1(i).

Lemma 4.4. The chain map $b$ as defined above induces the identity on all Milnor-Thurston homology groups $\mathcal{H}_{k}(X)$.

Proof. The established technical frame of showing this is to construct a chain homotopy $D_{n}: C_{n}(X) \rightarrow C_{n+1}(X)$ satisfying $\partial_{n+1} \circ D_{n}+D_{n-1} \circ \partial_{n}=$ $b_{n}-$ id. Theorem 2.1 can be used to extend the standard construction from singular homology theory to Milnor Thurston theory. For the sake of self-containedness we give the key-formulas for the definitions of $D_{n}$ in the following: For each proper subset $M_{\neq}^{\subset}\{0,1,2, \ldots, n\}$ and each permutation $\pi \in \mathcal{S}_{\# M}$ we first let

$$
\operatorname{sign}(M, \pi):=-\operatorname{sign}(\pi) \cdot(-1)^{\frac{\# M \cdot(\# M+1)}{2}} \cdot(-1) \sum_{m \in M} m .
$$

Then we construct the affine mapping $\gamma_{n, M, \pi}: \Delta_{n+1} \rightarrow \Delta_{n}$ by extending the definitions

$$
P_{j} \longmapsto \begin{cases}P & \text { for } j=0, \\ \widetilde{P}_{\chi_{M}(\pi(0)), \chi_{M}(\pi(1)), \ldots, \chi_{M}(\pi(j-1))} & \text { for } 1 \leq j \leq \# M, \\ P_{k} \text { where } k \text { is the }(j-\# M)^{\text {th }} & \\ \text { number of the totally ordered } & \\ \text { set }\{0, \ldots n\}-M & \text { for } \# M<j \leq n+1\end{cases}
$$

where $\chi_{M}$ is the strictly monotonously increasing mapping $\{0,1,2, \ldots, \# M-$ $1\} \rightarrow M$. Finally we define the chain $c_{\gamma, n} \in C_{n+1}\left(\Delta_{n}\right)$ as $\sum_{M, \pi} \operatorname{sign}(M, \pi)$. $\gamma_{n, M, \pi}$ and let $D_{n}: C_{n}(X) \rightarrow C_{n+1}(X), \sigma \mapsto \sigma_{\bullet}\left(c_{\gamma, n}\right)$. Analogously as in $4.2 / 4.3$, both parts of Theorem 2.1 can be applied to such a construction, which gives the result that the chain-homotopy also exists in MilnorThurston theory.

Lemma 4.5. Let $U \subset W \subset X$ satisfy $\bar{U} \subset \stackrel{\circ}{W}$. Then for any measure $\mu \in \mathcal{C}_{k}(X)$ there exists $r \in \mathbb{N}$ such that $b^{r}(\mu)=\nu_{1}+\nu_{2}$, where $\nu_{1}$ and $\nu_{2}$ are measures defined on $C^{0}\left(\Delta_{k}, X-U\right)$ and $C^{0}\left(\Delta_{k}, W\right)$, respectively.

Proof. Note that the sequence of diameters obtained by iterating the barycentric subdivision of an affine simplex tends to zero. Nevertheless an additional construction is required to get from this basic observation to a valid proof of the lemma: To wit, here singular simplices of a possibly non-metrizable space and especially the simultaneous barycentric subdivision of infinitely many singular simplices must be considered, namely of all simplices belonging to the quasicompact determination set of the measure.

Let $\mu$ be a measure $\in \mathcal{C}_{k}(X)$ and let $D$ be a quasicompact determination set of $\mu$. Consider

$$
F: D \times \Delta_{k} \rightarrow X, \quad(\sigma, x) \mapsto \sigma(x),
$$


which gives a continuous mapping. Hence $F^{-1}(\bar{U})$ and $F^{-1}(X-\stackrel{\circ}{W})$ are disjoint closed subsets of the set $D \times \Delta_{k}$ which is quasicompact by [vQ], (Satz 8.13), [Will], ${ }^{2}$ Chap. 6 (Thm 17.8). Let $\rho$ be the usual Euclidean metric which is defined on each fibre $\{\sigma\} \times \Delta_{k}$ and consider the function

$$
d: D \rightarrow \mathbb{R}
$$

$$
\sigma \mapsto \rho\left(F^{-1}(\bar{U}) \cap\left(\{\sigma\} \times \Delta_{k}\right), F^{-1}(X-\stackrel{\circ}{W}) \cap\left(\{\sigma\} \times \Delta_{k}\right)\right) .
$$

$d$ need not be continuous, but is "semicontinuous" in the sense that, given $\varepsilon>0, d\left(\sigma^{\prime}\right)>d(\sigma)-\varepsilon$ for all $\sigma^{\prime}$ that are contained in an appropriate neighbourhood of $\sigma$. This semicontinuity which is proved in the following proposition will be sufficient to complete this proof in 4.7 .

Proposition 4.6. $d$, as defined in 4.5(2), is semicontinuous.

Proof. Let

$$
E:=\left(\left(D \times \Delta_{k}\right)-\left(F^{-1}(\bar{U}) \cup F^{-1}(X-\stackrel{\circ}{W})\right)\right) \cap\left(\{\sigma\} \times \Delta_{k}\right),
$$

which is an open part of the fibre $\{\sigma\} \times \Delta_{k}$. Define open boundary strips of $E$, construct $E^{\prime}$ by removing these boundary strips from $E$ and define $\left(F^{-1}(\bar{U})\right)^{\prime}$ and $\left(F^{-1}(X-\stackrel{\circ}{W})\right)^{\prime}$ by adding these boundary strips to $\left(F^{-1}(\bar{U})\right) \cap\left(\{\sigma\} \times \Delta_{k}\right),\left(F^{-1}(X-\stackrel{\circ}{W})\right) \cap\left(\{\sigma\} \times \Delta_{k}\right)$ respectively. We obtain $\rho\left(\left(F^{-1}(\bar{U})\right)^{\prime}, \quad\left(F^{-1}(X-\stackrel{\circ}{W})\right)^{\prime}\right) \geq d(\sigma)-\varepsilon$. Now we want to construct an open neighbourhood of $E^{\prime}$ in product form that is still contained in $\left(D \times \Delta_{k}\right)-\left(F^{-1}(\bar{U}) \cup F^{-1}(X-\stackrel{\circ}{W})\right)$. Since the latter set is open, each point of $E^{\prime}$ can be surrounded by an open neighbourhood in product form that is still contained in that set. Because $E^{\prime}$ is a closed part of the compact fibre $\{\sigma\} \times \Delta_{k}$, finitely many of those small product neighbourhoods give a covering of $E^{\prime}$. If then we let $U(\sigma)$ be the intersection of the $D$-component of those finitely many product neighbourhoods and let $U\left(E^{\prime}\right)$ be the union of the corresponding $\Delta_{k}$-components, we get $E^{\prime} \subset U(\sigma) \times U\left(E^{\prime}\right) \subset\left(D \times \Delta_{k}\right)-\left(F^{-1}(\bar{U}) \cup F^{-1}(X-\stackrel{\circ}{W})\right)$. Hence $d(\sigma)-\varepsilon$ is a lower bound for the values taken by $d$ on $U(\sigma)$.

\subsection{Completion of the proof of 4.5.}

By combining this semicontinuity and the quasicompactness of $D$ with the fact, that $d$ has no zeros by construction, we get that it takes on a positive minimum. From this point of view the observation as mentioned in the first sentence of 4.5 is sufficient to conclude that there exist $r$ so that any simplex $\sigma$ obtained by $r$-fold barycentric subdivision of the simplices of $D$

\footnotetext{
${ }^{2}$ See footnote of 2.2
} 
satisfies either $\sigma\left(\Delta_{k}\right) \cap \bar{U}=\emptyset$ or $\sigma\left(\Delta_{k}\right) \cap(X-\stackrel{\circ}{W})=\emptyset$ or both of these. We fix one of those $r$ and let $\iota: C^{0}\left(\Delta_{k}, X\right) \rightarrow\{0,1\}$ be the indicator-function of $\left\{\sigma \in C^{0}\left(\Delta_{k}, X\right) \mid \sigma\left(\Delta_{k}\right) \cap \bar{U} \neq \emptyset\right\}$. Then $\nu_{1}:=\iota \cdot b^{r}(\mu)$ and $\nu_{2}:=(1-\iota) \cdot b^{r}(\mu)$ have all the desired properties.

4.8. Remark. Note that the preceding Lemma 4.5 does neither imply that $\nu_{1} \in \mathcal{C}_{k}(X-U)$ nor that $\nu_{2} \in \mathcal{C}_{k}(W)$, since for these measures we did not construct quasicompact determination sets contained in $C^{0}\left(\Delta_{k}, X-\right.$ $U$ ) and $C^{0}\left(\Delta_{k}, W\right)$, respectively. The following example shows that such determination sets may not exist even after repeated arbitrary barycentric subdivision. Hence this example implies that the usual statement of the excision theorem could not be obtained in Milnor-Thurston theory from the usual techniques based on barycentric subdivision alone.

4.9. Example. Let

$$
\begin{aligned}
& X=(\{(x, 1) \mid x \in[0,1]\} \cup\{(x, 0) \mid x \in[0,1]\}) / \sim \\
& \text { where }(x, 0) \sim(x, 1) \text { iff } x<\frac{1}{2}, \\
& W=\{(1,1)\} \cup\{(x, 0) \mid x \in[0,1]\} \subset X, \\
& U=\left\{(x, 0) \mid x \geq \frac{1}{2}\right\} \subset W .
\end{aligned}
$$

Consider the sets of line segments

$$
\begin{aligned}
& \left\{\overline{(x, 1)(1,1)} \mid x \leq \frac{1}{2}\right\}=L_{1}, \\
& \left\{\overline{(x, 0)(1,0)} \mid x \leq \frac{1}{2}\right\}=L_{2}
\end{aligned}
$$

as singular one-simplices by a natural parametrization. Let $\lambda_{i}(i \in\{1,2\})$ be the Lebesgue measure on $L_{i}$, which is defined according to the natural identification $L_{i} \leftrightarrow\left[0, \frac{1}{2}\right]$ via the parameter $x$. Finally let $\mu_{i}$ be the extension of $\lambda_{i}$ by zero to $C^{0}\left(\Delta_{1}, X\right)$ (cf. 1.11(1)). Then $\mu:=\mu_{1}-\mu_{2}$ is a relative cycle in $\mathcal{Z}_{1}(X, W)$, since $\partial(\mu)=+\frac{1}{2} \cdot(1,1)-\frac{1}{2} \cdot(1,0)$. Note that in this formula and below we identify the points with singular zero-simplices. Note further that when constructing $\partial \mu$ the points $(x, 0)$ cancel against $(x, 1)$ for $x<\frac{1}{2}$ because of " ", and, altough there is no cancellation of $\left(\frac{1}{2}, 1\right)$ against $\left(\frac{1}{2}, 0\right)$ we do not obtain an additional summand in the formula of $\partial \mu$, since the Lebesgue measure of a single point is zero. If we want to split up $\mu=\nu_{1}+\nu_{2}$ according to 4.5 there is only one choice: $\nu_{1}:=\mu_{1}, \nu_{2}:=-\mu_{2}$. But $\nu_{1}$ is not a relative cycle any more: We have $\partial \nu_{1}=\frac{1}{2} \cdot(1,1)-\left.\lambda\right|_{\left[0, \frac{1}{2}\right]}$, where $\lambda$ denotes the Lebesgue measure. Now a determination set of $\left.\lambda\right|_{\left[0, \frac{1}{2}\right]}$ must not contain any of the points $\left(\frac{1}{2}, 1\right)$ or $\left(\frac{1}{2}, 0\right)$, since $\left(\frac{1}{2}, 1\right) \notin W$ and $\left(\frac{1}{2}, 0\right) \in U$. On the other hand, no (quasi)compact subset of $\left[0, \frac{1}{2}\right)$ has the full Lebesgue measure, and thus it is not a determination set. Hence there is no decomposition of $\mu$ as 
required in 4.10 and by a finitely iterated barycentric subdivision, i.e. by replacing $\mu$ by $b^{r}(\mu)$ we get no principal differences in our situation.

After having seen this technical obstruction for a more generalized statement, we continue the proof of the excision theorem under our weaker assumptions:

Lemma 4.10. If in addition to the assumptions of 4.5 there exists $V$ as required in 4.1 , we get $\nu_{1} \in \mathcal{C}_{k}(X-U)$ and $\nu_{2} \in \mathcal{C}_{k}(W)$ for appropriate $r$.

Proof. As immediate consequence of the compact-open topology we get that $\left\{\sigma \in C^{0}\left(\Delta_{k}, X\right) \mid \sigma\left(\Delta_{k}\right) \subset O\right\}$ is an open subset of $C^{0}\left(\Delta_{k}, X\right)$, if $O \stackrel{\text { open }}{\subset} X$. As complements of such sets we have that $\left\{\sigma \in C^{0}\left(\Delta_{k}, X\right) \mid \sigma\left(\Delta_{k}\right) \cap \bar{V} \neq \emptyset\right\}$ and $\left\{\sigma \in C^{0}\left(\Delta_{k}, X\right) \mid \sigma\left(\Delta_{k}\right) \cap(X-\stackrel{\circ}{V}) \neq \emptyset\right\}$ are closed. Since closed subsets of quasicompact sets are always quasicompact $\left([\mathbf{v Q}]\right.$, (Satz 8.4)/[Will],$^{3}$ Chap. 6 (Thm 17.5a)) the intersection of a quasicompact determination set with any of these sets always gives a quasicompact set again. Now choose, similar as in 4.7, $r$ so big, that each singular simplex obtained from $r$-fold barycentric subdivision of those singular simplices $\sigma: \Delta_{k} \rightarrow X$ contained in the determination set $D$ cannot take on values in both sets $\bar{V}$ and $X-\stackrel{\circ}{W}$, and cannot take on values in both sets $\bar{U}$ and $X-\stackrel{\circ}{V}$. We then have to adapt the definitions of $\nu_{1}$ and $\nu_{2}$ as in 4.5/4.7 slightly by now letting $\iota$ to be the indicator function of $\left\{\sigma \in C^{0}\left(\Delta_{k}, X\right) \mid \sigma\left(\Delta_{k}\right) \cap \bar{V} \neq \emptyset\right\}$ and by then using the same equations $\nu_{1}=\iota \cdot b^{r}(\mu), \nu_{2}=(1-\iota) \cdot b^{r}(\mu)$ as in 4.5. However, now $D_{1}=\left\{\sigma \in D \mid \sigma\left(\Delta_{k}\right) \cap \bar{V} \neq \emptyset\right\}$ and $D_{2}=\left\{\sigma \in D \mid \sigma\left(\Delta_{k}\right) \cap(X-\stackrel{\circ}{V}) \neq \emptyset\right\}$ give quasicompact determination sets for $\nu_{1}$ and $\nu_{2}$, respectively.

\subsection{Completion of the proof of the excision Theorem 4.1.}

The proof now follows the standard stategy: The technical goal of the proof is to show that the homomorphism $\mathcal{H}_{k}(X-U, W-U) \rightarrow \mathcal{H}_{k}(X, W)$, which is naturally induced by the inclusion $(X-U, W-U) \subset(X, W)$, is bijective. The surjectivity follows, since $\nu_{1}$, as constructed in 4.5/4.10, satisfies $\mu \sim b^{r}(\mu)=\nu_{1}+\nu_{2}$ and hence can be regarded as preimage of $\mu$ according to this homomorphism induced by the natural inclusion. The injectivity follows by applying a similar argument to a $(k+1)$-dimensional chain which is assumed to provide the homology for two $k$-dimensional chains on the $(X, W)$-level, but not on the $(X-U, W-U)$-level.

4.12. Remark. Roughly speaking, the main construction that gives the proof of the excision theorem is to project a relative $(X, W)$-cycle on its $(X-U, W-U)$-component after a finitely iterated barycentric subdivision. Even if barycentric subdivision turns out to be a continuous process, the

\footnotetext{
${ }^{3}$ See footnote of 2.2
} 
projection is not a continuous map. This fact might be understood as the key reason for the difficulties described in this section (cf. Introduction). However, it will remain the only place of non-continuity that we will come upon in the course of tracing the arguments of standard homology theory within the scope of this paper.

\section{The Coincidence Proof.}

The whole section is devoted to this proof. We start by stating the corresponding result precisely:

The coincidence theorem 5.0. Let $X$ be a topological space that can be triangulated as simplicial complex and let $Y$ be a subspace which can be realized as subcomplex according to some triangulation of $X$. Then $\eta$ as defined in 5.1(1) induces natural isomorphisms for the homology groups $H_{k}(X) \stackrel{\cong}{\rightrightarrows} \mathcal{H}_{k}(X)$ and $H_{k}(X, Y) \stackrel{\cong}{\rightrightarrows} \mathcal{H}_{k}(X, Y)$.

As already mentioned in the Introduction, the outline of this proof is due to Eilenberg and Steenrod, who aimed to present such a coincidence theory in a completely abstract way ([ES], III.10.1). However in the sequel we rather follow the presentation of this matrial in Spanier's book ( $[\mathbf{S p}]$, 4.8.10, but cf. also [ES], VII.§10). The following paragraphs correspond to the steps of this proof.

5.1. Step one. Definition of a canonical chain map $C_{*}(X) \rightarrow \mathcal{C}_{*}(X)$ : Take

$$
\eta_{k}: \sum_{i} \alpha_{i} \cdot \sigma_{i} \longmapsto \sum_{i} \alpha_{i} \cdot \mu_{\sigma_{i}} \quad \forall k \in \mathbb{N}
$$

where $\alpha_{i} \in \mathbb{R}$, the $\sigma_{i}$ are $k$-dimensional singular simplices and $\mu_{\sigma_{i}}$ denotes atomic measures, cf. 1.4(i). In order to see that this construction gives a chain map, recall that (cf. 2.9) the boundary operator in Milnor-Thurston homology is defined by using essentially the same formula as in singular homology. Hence the processes of replacing all simplices by their boundary chains and of replacing simplices with the corresponding atomic measure naturally commute, i.e. we have $\eta_{k-1} \circ \partial_{k}=\partial_{k} \circ \eta_{k}$ for all $k$. This means, that all $\eta_{k}$ together form a chain map inducing homomorphisms on the homology groups. The purpose of the forthcoming steps is to show that these induced homomorphims $\left(\eta_{*}\right)_{k}$ all are isomorphisms.

\subsection{Step two. Verifying the Exactness Axiom:}

Recall that relative Milnor-Thurston chain groups were obtained as quotient groups. Thus, if $\mathcal{C}_{*}$ is used to denote a chain complex, we have a short exact sequence of chain complexes for each $A \subset X$ :

$$
0 \longrightarrow \mathcal{C}_{*}(A) \longrightarrow \mathcal{C}_{*}(X) \longrightarrow \mathcal{C}_{*}(X, A) \longrightarrow 0 .
$$


By standard arguments of homological algebra (e.g. see [StZ], III.8.3.8 and [StZ], III.7.4.5 or [Sp], 4.5.4) such a sequence induces a long exact homology sequence.

5.3. Step three. Verifying the Homotopy Invariance:

(1) Absolute Case. Originally, in order to obtain the statements 2.10(vi)/

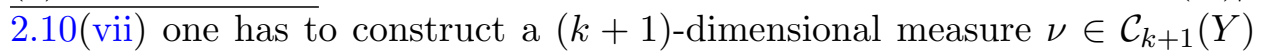
such that $\partial \nu=f(\mu)-g(\mu)$, where $f \simeq g: X \rightarrow Y$ and a measure-cycle $\mu \in \mathcal{Z}_{k}(X)$ are given. The established technical frame (cf. [StZ], III.9.3.1 and [StZ], III.9.3.7-III.9.3.8 or [Sp], 4.4.9) of doing so is to prove that $f_{\bullet}$ and $g_{\bullet}$ are chain homotopic by proving the existence of some chain homotopy $\left(D_{k}\right)_{k \in \mathbb{N}}$ satisfying $\left(D_{k-1} \circ \partial_{k}\right)\left(\sigma_{k}\right)+\left(\partial_{k+1} \circ D_{k}\right)\left(\sigma_{k}\right)=f_{\bullet}\left(\sigma_{k}\right)-g_{\bullet}\left(\sigma_{k}\right)$. Since in Milnor-Thurston homology we can stick to this frame, similarly as in 4.4 we have merely to show that the definition of the $D_{k}$-morphisms extends compatibly with the above properties to Milnor-Thurston theory. In singular homology theory the standard definition of the $D_{k}$-mappings is given by the following formula

$$
C_{k}(X) \ni \sigma_{k} \stackrel{D_{k}}{\longmapsto}\left(H_{\bullet} \circ\left(\sigma_{k} \times \operatorname{id}_{I}\right)_{\bullet}\right)\left(c_{k+1}\right) \in C_{k+1}(Y)
$$

where $H$ denotes the homotopy between $f$ and $g$ and is regarded as a mapping $X \times I \rightarrow Y$, and $c_{k+1}$ denotes a fixed chain of $C_{k+1}\left(\Delta_{k} \times I\right)$. This chain is to be chosen in such a way that $\partial c_{k+1}$ contains (with opposite signs) the singular simplexes $\Delta_{k} \rightarrow \Delta_{k} \times\{0\}$ and $\Delta_{k} \rightarrow \Delta_{k} \times\{1\}$ and that the remaining summands of $\partial c_{k+1}$ give some triangulation of $\left(\partial \Delta_{k}\right) \times I$, i.e. give the same $k$-chain in $\left(\partial \Delta_{k}\right) \times I$ which also could be obtained by $\sum_{i=0}^{k}(-1)^{i}\left(\delta_{i} \times \operatorname{id}_{I}\right) \bullet\left(c_{k}\right)$ where $\delta_{i}: \Delta_{k-1} \rightarrow \Delta_{k}$ has been defined in 2.9(1).

Such a chain may either be constructed by an explicit triangulation of the prisma $\Delta_{k} \times I$ or it can be obtained by an arbitrary choice in the course of an induction over $k$ using the acyclicity of $\Delta_{k} \times I$. But even in the latter case this arbitrary choice does not provide any obstruction to the continuity of this definition, since in each dimension $k$ only one chain in the space $\Delta_{k} \times I$ is chosen and this fixed chain is used in the definition of $D_{k}$ for each singular simplex $\sigma_{k}$.

By replacing $c_{k+1}$ by its representation as a linear combination $\sum_{j=1}^{m} \alpha_{j} \cdot \tau_{j}$ of singular simplices $\tau_{j} \in C_{k+1}\left(\Delta_{k} \times I\right)$ one can use the homomorphismproperties of the chain maps ()• to obtain the following alternative description of the $D_{k}$-mappings:

$$
C_{k}(X) \ni \sigma_{k} \stackrel{D_{k}}{\longmapsto} \sum_{j=1}^{m} \alpha_{j} \cdot\left(H_{\bullet} \circ\left(\sigma_{k} \times \mathrm{id}_{I}\right)_{\bullet}\right)\left(\tau_{j}\right) \in C_{k+1}(Y) .
$$

If one recalls the definition of the chain maps $H_{\bullet}$ and $\left(\sigma_{k} \times \mathrm{id}_{I}\right)_{\bullet}$, one gets that the essential definition of the summands of $D_{k}$ can be viewed as follows:

$$
\sigma_{k} \longmapsto H \circ\left(\sigma_{k} \times \mathrm{id}_{I}\right) \circ \tau_{j} .
$$


This mapping is continuous, because it is a product of the mappings considered in 2.8(i)-2.8(iii). Now from 2.1 it follows that the definition of the homomorphisms $D_{k}$ can be extended to Milnor-Thurston theory such that the extensions also satisfy the properties of a chain homotopy. Then one gets $f_{*}=g_{*}$ by standard conclusions of homological algebra (see [StZ], III.8.3.12-III.8.3.13 or [Sp], 4.2.2 and cf. 2.10(vi)-(viii)).

(2) Relative Case. The treatment of the relative case is essentially the same as in the absolute case. Note that the main difference between these two statements is that in the relative case we have to consider relative cycles, whilst the absolute case is a statement about absolute cycles, only. Further note (e.g. cf. 4.8/4.9) that in some situations skipping to the relative case can cause difficulties in Milnor-Thurston homology theory, since having a relative cycle $z_{k} \in \mathcal{Z}_{k}(X, A)$ always implies that there has to exist a quasicompact determination set for $\partial z^{k}$ in $A$ which may not exist in any context. However, in this situation here, where the assumptions of the relative case are that $f$ and $g$ are homotopic maps $X \rightarrow Y$ satisfying that for some subspaces $A \subset X$ and $B \subset Y$ we have $f(A) \subset B, g(A) \subset B$ and that the homotopy $H$ respects the subspace structure, i.e. that $H(A \times I) \subset B$, the existence of all the determination sets does follow from standard image measure constructions. Since the assumptions for $f$ and $g$ are not weaker, but stronger in the relative case, in fact the same chain homotopy construction as in the absolute case gives the desired result here.

\subsection{Step four. Verifying the Initial Axiom.}

There are no obstructions for an elementary calculation of the homology groups of a space with a single point: Since such a space ("*") permits the definition of one and only one singular simplex in each dimension (namely the constant mapping), we get for each real number one measure on $C^{0}\left(\Delta_{k}, *\right)$. This situation is completely analogous to the case of singular homology theory, giving the same results (cf. [StZ], III.9.1.10).

\subsection{Step five. Verifying the Excision Axiom.}

Section four was devoted to this step. Note that the additional assumption of the existence of $V$ in 4.1 does not matter in the present context, since it is automatically fulfilled by simplicial complexes because they are normal (cf. [Dug], VII.3.2(2)).

5.6. Step six is the standard conclusion due to Eilenberg and Steenrod proving the coincidence of the homology groups based on the properties 5.1-5.5 for finite simplicial complexes. It is, roughly speaking, a kind of induction proof showing that there exists no smallest simplicial complex with non-coinciding homology groups by comparing a simplicial complex with what is obtained by removing one highest dimensional simplex. More technically speaking, we induct over all pairs $(K, L)$ of simplicial complexes 
by considering the number $k$ of simplices in the complex $K$. The case $k=1$ has to be considered as zero-step of the induction. If a simplicial complex consists of one simplex only, it has to be a zero-dimensional one, and hence there are two pairs of simplicial complexes (namely $(*, \emptyset)$ and $(*, *)$ ) which only have to be considered in the initial step. In case $(*, *)$ in both theories of consideration all relative chain groups are zero, and thus all homology groups coincide in being zero also. The zero-step of our induction follows since the case $(*, \emptyset)$ had been discussed in 5.4. The inductive step now is based on comparing the homology groups of the simplicial complex with those of the complex with one highest dimensional simplex removed by using 5.2, 5.3 and 5.5. For the sake of completeness, we include an outline of this proof:

Consider the system of commutative diagrams obtained by taking the long exact homology sequences of both homology theories under consideration and what is induced by the natural chain mapping $\eta$ :

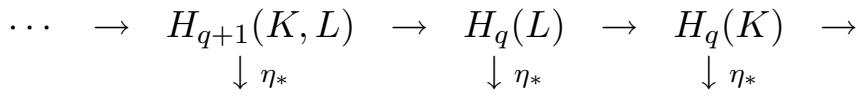

$$
\begin{aligned}
& \cdots \rightarrow \mathcal{H}_{q+1}(K, L) \rightarrow \mathcal{H}_{q}(L) \rightarrow \mathcal{H}_{q}(K) \rightarrow
\end{aligned}
$$

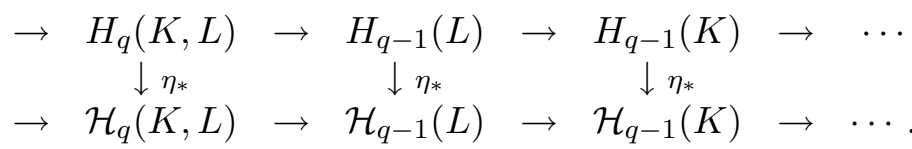

Then the desired conclusion follows by applying the Five Lemma (cf. [StZ], III.8.2.3 or [Sp], 4.5.11 or [ES], I.4.3) twice to this diagram. Assume that the complex $K$ has $k+1$ simplexes, which is one simplex more as we may assume by the induction hypothesis that $\eta_{*}$ must be an isomorphism; and choose as subcomplex $L$ for the first application $L=\Delta$, where $\Delta$ is one highest dimensional simplex and $P$ is used to denote its barycentre. Then we have that all vertical arrows in (1) which correspond to the subcomplex are isomorphisms due to the induction hypothesis, and the same for all vertical arrows related to the relative groups, since by applying the excision axiom and the homotopy invariance we get that

$$
\mathcal{H}_{q}(K, \Delta) \stackrel{5.5 / 4.1}{=} \mathcal{H}_{q}(K-\{P\}, \Delta-\{P\}) \stackrel{5.3}{=} \mathcal{H}_{q}(K-\stackrel{\circ}{\Delta}, \partial \Delta)
$$

reducing the situation to a complex with one simplex less. Then the Five Lemma gives that the vertical arrows corresponding to $K$ are also isomorphisms, and hence we obtain coincidence for all absolute homology groups of all complexes with at most $k+1$ simplices. Then we consider again the diagram (1) for an arbitrary complex $K$ with $k+1$ simplices, and at this time for an arbitrary subcomplex $L$ of $K$. Now we have that the vertical arrows related to $K$ and to $L$ are isomorphisms, and hence the Five Lemma 
gives the same for all relative groups, also. This completes the induction argument.

5.7. Step seven. Showing the coincidence for infinite simplicial complexes also.

Observe that the image of each measure (cf. 1.7) is only contained in a finite number of simplexes in any case, because it can be regarded as a quasicompact set $F\left(D \times \Delta_{k}\right)$, cf. $4.5(1)$. Assume that $\eta_{*}: H_{k}(K) \rightarrow \mathcal{H}_{k}(K)$ is not an isomorphism and construct the finite subcomplex $L$ of $K$ containing the image of a "violating measure". Such a violating measure is, in the case when $\eta_{*}$ is not onto, a measure which has no preimage with respect to $\eta_{*}$, and in the case when $\eta_{*}$ is not one-to-one, it is a $(k+1)$-dimensional measure whose boundary is the image of a non-nullhomotopic cycle $\in Z_{k}(K)$. Then from considering the commutative diagram

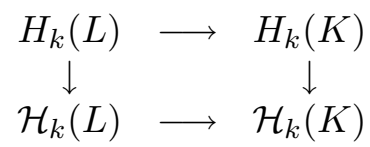

the desired contradiction follows staightforwardly in both cases.

\section{The Non-Coincidence Example.}

The purpose of this section is to define a space $L$ and a measure $\mu$ on it such that $\mu$ is a non-nullhomotopic cycle in $\mathcal{Z}_{1}(L)$ and such that it is not contained in $\eta_{*}\left(H_{1}(L)\right)$ (cf. 5.1). The desired space $L$ is obtained by choosing $P_{1}, P_{2} \in \mathbb{R}^{2}$ arbitrarily, and by connecting them on the one hand by a line segment $l_{0}$ and on the other hand by a system of countably many circle segments $\left(l_{i}\right)_{i \in \mathbb{N}}$ as pictured in Fig. a. Then $L:=\bigcup_{i=0}^{\infty} l_{i}$, i.e. $L$ is defined as the union of all those segments and topologized with the relative topology obtained from its embedding into $\mathbb{R}^{2}$.

Note that each of the $\ell_{i}$ can be regarded as a singular 1 -simplex. This system of 1-simplices is compact, because it is a union of a convergent sequence with its limit. Then

$$
\mu:=-\mu_{\ell_{0}}+\sum_{i=1}^{\infty} \frac{1}{2^{i}} \cdot \mu_{\ell_{i}}
$$

is an infinite counting measure (cf. 1.4(ii)) that is a well-defined cycle in $\mathcal{Z}_{1}(L)$. However, it is not nullhomologous as can be seen by considering its image $i_{\bullet}(\mu)=i(\mu)$ in $L \cup D_{2}$, where $D_{2}$ is the subdisk of $\mathbb{R}^{2}$ bounded by $l_{0}$ and $l_{2}$ and $i$ is the canonical embedding $L \hookrightarrow L \cup D_{2}$. The latter space is free of accumulation phenomena, and hence, unlike $L$, it is a simplicial complex. Thus the coincidence theorem 5.0 can be used to conclude that $i(\mu) \sim \frac{1}{2} \cdot \mu_{\ell_{1}}-\frac{1}{2} \cdot \mu_{\ell_{0}} \nsim 0$, hence $\mu \nsim 0$ itself. 


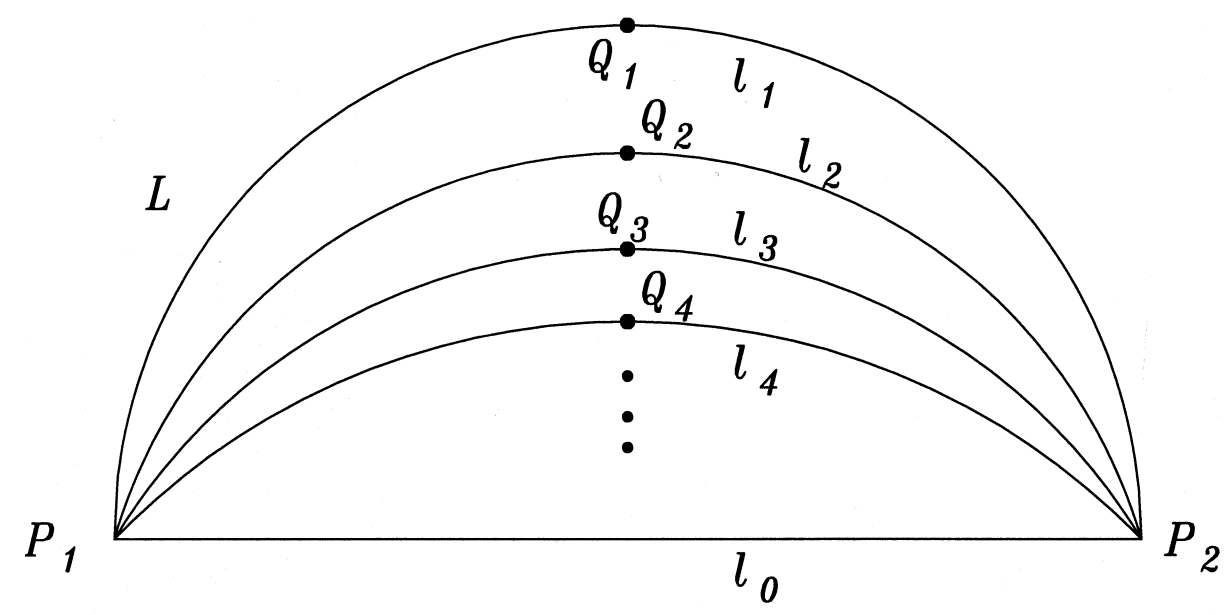

Fig. a:

This figure shows a picture of the space $L$ on which the measure $\mu$ giving the desired counter-example to the validity of a coincidence theorem for all topological spaces is defined.

By similarly applying Theorem 5.0 to the (infinitely) triangulable space $\left(L \cup D_{2}\right)-\left\{Q_{1}\right\}$, we get that any measure $\mu^{\prime} \in \mathcal{Z}_{1}\left(L \cup D_{2}\right)$ that does not contain $Q_{1}$ in its (unique, cf. 3.3) image can only represent nullhomotopic cycles. Hence such a measure cannot be homologous to $\mu$ in $\mathcal{H}_{1}(L)$. The above result can easily be extended to any $Q_{j}$ by considering instead of $D_{2}$ some other disk that spans all the $l_{i}$ apart from $l_{j}$. For each $j \geq 1$ such a disk can easily be constructed, at least when $L$ is embedded into $\mathbb{R}^{3}$, and hence we get that any $\mu^{\prime}$ which is homologous to $\mu$ has to contain all the $Q_{j}$ in its image. The latter property cannot be satisfied by a finite counting measure, since no singular one-simplex, i.e. no path in $L$ defined on a compact interval, may contain infinitely many of the points $Q_{j}$ without violating continuity. Hence $\mu$ cannot be homologous to a finite counting measure, and therefore the homology class that is represented by $\mu$ is not contained in $\eta_{*}\left(H_{1}(L)\right)$. Thus $\eta_{*}$ is not onto, and hence $H_{1}(L)$ and $\mathcal{H}_{1}(L)$ are not canonically isomorphic.

Remark. With more effort we could have also proved that these groups are not even isomorphic, since $H_{1}(L)$ is a countable-dimensional real vector space, whereas $\mathcal{H}_{1}(L)$ is of uncountable dimension. 


\section{References}

[Bau1] H. Bauer, Wahrscheinlichkeitstheorie und Grundzüge der Maßtheorie, deGruyer, Berlin-New York, 2.Auflage 1974;

or, in its first English Translation: 'Probality Theory and Elements of measure Theory $^{\prime 4}$, Series in quantitative methods of decision making, Holt Rinehard and Winston, New York and London, 1972.

Revised English Translation within the series 'Probability and Mathematical Statistics', Academic Press, London, 1981.

[Bau2] _ Maß- und Integrationstheorie, deGruyer, Berlin-New York, 1990.

[BP] R. Benedetti and C. Petronio, Lectures on Hyperbolic Geometry, Universitext, Springer-Verlag, Berlin-Heidelberg-New York, 1992.

[Dug] J. Dugundji, Topology, Allyn and Bacon Series in Advanced Mathematics, Allyn and Bacon Inc., Boston, 1966.

[ES] S. Eilenberg and N. Steenrod, Foundations of Algebraic Topology, Princeton Mathematical Series No. 15, Princeton University Press, Princeton (New Jersey), 1952.

[Gr] M. Gromov, Volume and bounded cohomology, Publ. Math. I.H.E.S., 56 (1982), 5-99.

[HM] U. Haagerup and H.J. Munkholm, Simplices of maximal volume in hyperbolic n-space, Acta Math., 147 (1981), 1-11.

[Hal] P.R. Halmos, Measure Theory, The University Series in Higher Mathematics, van Nostrand, New York, 1950.

Reprint as: Graduate Texts in Higher Mathematics No. 18, Springer-Verlag, New York-Heidelberg-Berlin, 1974.

[Hen] E. Henze, Einführung in die Maßtheorie, B.I.-Hochschultaschenbücher Bd. 505, Bibliographisches Institut, Mannheim, 1971.

[MR] Mathematical Reviews, issued by the American Mathematical Society, Providence, Rhode Island, USA.

[MTh] J. Milnor and W.P. Thurston, Characteristic numbers of three-manifolds, Enseign. Math. II, Ser. 23 (1977), 249-254.

[Mnkh] H.J. Munkholm, Simplices of Maximal Volume in Hyperbolic Space, Gromov's Norm, and Gromov's Proof of Mostow's Rigidity Theorem (following Thurston) in: Koschorke, Ulrich and Neumann, Walter D. (eds.), 'Topology Symposium Siegen 1979', Procedings of a Symposium held at the University of Siegen June 14-19, 1979, Lecture Notes in Mathematics No. 788, Springer-Verlag, Berlin-HeidelbergNew York, 1980, 109-124.

[Mnkr] J.R. Munkres, Elementary differential topology, Annals of Mathematics studies No. 54, Princeton University Press, Princeton (New Jersey), revised edition 1966.

[vQ] B. von Querenburg, Mengentheoretische Topologie, Hochschultext, SpringerVerlag, Berlin-Heidelberg-New York, 2.Auflage 1979.

[Rcl] J.G. Ratcliffe, Foundations of Hyperbolic Manifolds, Graduate Texts in Mathematics No. 149, Springer-Verlag, New York-Heidelberg-Berlin, 1994.

\footnotetext{
${ }^{4}$ Note that the German and the English editions of that book have slightly different enumeration systems. We are using the reference numbers of the second German edition when quoting from this book.
} 
[Sp] E.H. Spanier, Algebraic Topology, McGraw-Hill Series in Higher Mathematics, McGraw-Hill, New York, 1966.

[StZ] R. Stöcker und H. Zieschang, Algebraische Topologie, Mathematische Leitfäden, Teubner, Stuttgart, 1988.

[Th] W.P. Thurston, Geometry and Topology of Three-manifolds, circulated lecture notes, 1977/1978.

[Will] S. Willard, General Topology, Addison-Wesley Series in Mathematics, AddisonWesley, Reading (Massachusetts), 1970.

Received November 13, 1995 and revised April 10, 1997. Research for this paper was done in Bochum, Germany (the author's home address), in Toulouse, France and in Warwick, England. The contacts with Toulouse were supported by the Deutsche Forschungsgemeinschaft within the PROCOPE-Framework; the author's two-year-leave to Warwick was supported by the European Community within the 'Human Capital and Mobility'Programme of the European Community, No. ERBCHBICT930859.

FAKUltät UND Institut FÜR MAThEMATIK

RUHR-UNIVERSITÄT BOCHUM

D-44780 BOCHUM

GERMANY

E-mail address: az@maths.warwick.ac.uk 